\title{
LA SYSTÉMIQUE POUR COMPRENDRE LES CRISES DU SYSTÈME ÉDUCATIF FRANÇAIS
}

\author{
Author(s) / Auteur(s) : \\ Véronique GIGNOUX-EZRATTY \\ AFSCET \\ veronique.ezratty@cefasp.org
}

\begin{abstract}
Résumé :
Le système éducatif français est régulièrement secoué par des crises. Depuis 1975 et l'instauration pour les jeunes de 11 à 15 ans du collège unique avec des classes hétérogènes, l'Education nationale n'a jamais su trouver un fonctionnement satisfaisant permettant à tous les enfants de progresser sereinement. Les jeunes qui entrent au collège avec des lacunes en lecture et en expression écrite progressent peu et dans la majorité des cas ne rattrapent pas leur retard.

Les insatisfactions liées à cette inefficacité du système éducatif provoquent des crises régulières : révoltes sporadiques mais répétées dans les quartiers populaires, montée des comportements extrémistes chez les jeunes, interrogation des intellectuels et des dirigeants devant les médiocres résultats dans les enquêtes internationales sur la performance de l'école, crise du recrutement chez les enseignants.

La communication documentera les symptômes à partir d'enquêtes publiées, et avec les outils de la systémique, proposera des analyses des mécanismes à l'origine de ces crises. L'auteur attribue le blocage à des jeux de pouvoir entre quatre courants de pensée chez les personnes impliquées dans l'Education nationale.

Il sera mis en évidence de l'émergence, depuis 2014, d'une communauté de personnes investies qui partagent leurs analyses sur Internet, créant une culture commune qui dépasse les partis politiques et une évolution des paradigmes dominants en France sur l'éducation.
\end{abstract}

Keywords / Mots-clés :

système, education, crise, France

\section{INTRODUCTION}

Depuis la fin du XIXe siècle et la Troisième République, la France affiche une volonté de rendre le savoir accessible à tous. En 1882, les lois Ferry décrétaient l'instruction obligatoire pour les garçons et les filles entre 6 et 13 ans, dans les écoles primaires déjà gratuites. La scolarité obligatoire est passée à 16 ans en 1959. Les trois types d'enseignement qui existaient pour les jeunes entre 11 et 15 ans ont fusionné lors de la loi Haby de 1975 qui institua le même enseignement pour tous et les classes hétérogènes. C'est le « collège unique ». En 1984, la loi Savary supprima la sélection à l'entrée de l'université rendant cette dernière accessible aux jeunes hommes et jeunes filles ayant un baccalauréat, y compris aux titulaires d'un baccalauréat professionnel pour lequel les exigences en compétences littéraires sont moindres. Puis en 1985, le gouvernement afficha la volonté d'arriver à $80 \%$ de la population au niveau baccalauréat ${ }^{1}$.

Cependant, l'égalité d'accès aux enseignements n'a jamais été accompagnée par une égalité des possibilités de réussite. Environ $20 \%$ des élèves sortent de l'école primaire en maîtrisant insuffisamment la lecture et l'expression. Ces élèves progressent peu au collège qu'ils fréquentent entre 11 et 15 ans, car la France n'a jamais mis en place une organisation et un type d'enseignement qui permettent d'accompagner efficacement les élèves à besoins spécifiques. En particulier, les élèves qui ont des lacunes en expression, quelle qu'en soit la cause, sont le plus souvent en situation d'échec sans l'aide nécessaire pour combler leurs lacunes. De nombreux biais existent dans l'orientation qui fonctionne par l'élimination progressif sur le niveau en Français jusqu'à l'accès aux filières générales

\footnotetext{
${ }^{1}$ Le Baccalauréat est le diplôme de fin d'études secondaires en France qui se passe normalement à 18 ans.
} 
pour $40 \%$ d'entre eux. L'affectation dans les filières du lycée professionnel ne prend pas assez en compte la motivation de l'élève pour les métiers proposés et beaucoup trop les notes. L'accès à une formation n'implique pas le soutien pour que les élèves ayant de fortes lacunes aient une chance raisonnable d'y réussir.

L'écart entre les promesses et la réalité ainsi que les dénis de l'institution qui souvent l'accompagnent, ont créé des tensions qui provoquent régulièrement des crises. Les tentatives de réformes qui ont fait suite aux crises n'ont, jusqu'à maintenant, jamais produit les effets escomptés.

Cette communication attribue ce blocage aux jeux de pouvoir de quatre courants à l'intérieur du groupe social formé par les parties prenantes du système éducatif. Ces personnes interagissent pour construire des représentations de l'école plus ou moins partagées et donc forment un système social complexe. Dans ce système, il est possible de repérer quatre sous-systèmes qui imposent, plus ou moins consciemment, pour des raisons d'intérêts particuliers, des dogmes faux. Ces quatre courants sont : la « technostructure de l'Education Nationale », les « autoproclamés-progressifs », les « élitistes paradoxaux » et les « élitistes assumés ».

Un changement de paradigme est en cours dans la société civile responsable. Celui-ci a été possible grâce aux possibilités d'échanger en réseaux sur Internet. Des personnes ont fait le choix, anonymement ou non, de transgresser des tabous imposés par les courants. Elles ont publié des analyses pour donner une compréhension du système. Elles ont envoyé sur les réseaux sociaux ouverts des brefs ou longs messages sur la réalité des établissements scolaires ou sur le management pratiqué dans l'Education nationale. La réflexion a été décuplée par la concertation qui a précédé la loi de refondation de l'école de 2013, et les polémiques sur la réforme du collège de 2015 . Il est en train de se construire une compréhension commune des enjeux de l'école et des leviers pour une meilleure efficacité dont les fondamentaux transcendent les clivages politiques. Des dogmes bloquants sont peu à peu abandonnés.

Cependant, l'école Française n'a pas encore trouvé de solutions à toutes ses contradictions. La prise de conscience doit se poursuivre pour éviter des retours en arrière qui pourraient alimenter les tentations populistes.

\section{LES DYSFONCTIONNEMENTS SYSTEMIQUES DE L'EDUCATION EN FRANCE EN 2012}

Le système éducation en France a des dysfonctionnements connus depuis longtemps par une poignée de spécialistes qui agissaient à leur niveau. Cependant, en 2012, date du début du travail collective pour la refondation de l'école, la société civile impliquée n'avait pas collectivement conscience de ces dysfonctionnements.

Pour cette communication, la société civile impliquée sont les personnes que l'on retrouve dans les groupes de réflexion, les sociétés savantes ainsi que dans les syndicats et associations concernées par l'Education. Ces sont les moteurs des changements : il ne pourra y avoir un changement dans la durée sans une prise de conscience de la société civile impliquée qui interviendra au moment des mutations pour donner les arguments qui permettront de surmonter les blocages. Ce sont les régulateurs du système. Ils limitent les changements pas forcément bénéfiques, dictés par des besoins de communication d'un ministère.

Une des caractéristiques de la France est l'incapacité de diffuser des analyses simples qui devraient être évidentes pour la majorité des personnes constituant la société civile impliquée.

Ce paragraphe va décrire trois dysfonctionnements majeurs qui existaient en 2012 depuis plusieurs dizaines d'années et qui peuvent être expliqués simplement. Ils vont servir de base à la description de l'impact des crises dans les évolutions du système éducation en France. 


\section{La faiblesse d'attention stratégique pour les élèves à besoins spécifiques}

Jusqu'en 2012, la France se caractérisait par une faiblesse d'attention par le système global et sa gouvernance pour les élèves ayant des difficultés particulières d'apprentissage ou ayant des besoins spécifiques. La plupart des enseignants n'ont pas reçu de formation pour apprendre aux élèves ayant des fonctionnements cognitifs différents (dyslexie, dysphasie, autisme, surdouance..), ni pour enseigner à des enfants qui ne maîtrisent pas la langue, ni pour aider les enfants qui font des blocages devant les apprentissages. Or, la loi du 11 février 2005 pour l'égalité des droits et des chances, la participation de la citoyenneté des personnes handicapées prévoit que le service public d'éducation prend en charge l'inclusion scolaire de tous les enfants. Ce n'est toujours pas le cas, les AESH (accompagnants des élèves en situation de Handicap) n'étant pas assez nombreux.

Le programme est divisé en cycles. Aussi, si un enfant n'a pas acquis les automatismes de la lecture avec construction de sens en fin de CE2 ( 9 ans) fin du cycle 2, les moyens ne sont pas en place pour qu'il ou elle puisse travailler par la suite cette compétence fondamentale. C'est le même problème pour le calcul. Différentes études montrent que $20 \%$ des élèves en fin de $6^{\mathrm{e}}$ n'ont pas acquis les mécanismes de lecture avec construction automatique de sens ${ }^{2}$.

Et pourtant le niveau des Français en lecture en fin de CM1 (10 ans) baisse dans les études internationales. Dans l'étude PIRLS 2016 qui mesure les performances en compréhension de l'écrit des élèves en fin de l'équivalent du CM1 (10 ans), la France se situe dans les plus faibles de l'Europe et les scores baissent depuis la première étude de 2001.

\section{L'abandon structurel au collège de celles et ceux qui n'ont pas les acquis en lecture}

Le collège est la période qui va de 11 à 15 ans, de la classe de $6^{\mathrm{e}}$ à la classe de $3^{\mathrm{e}}$ dans le système scolaire français.

Le collège tel qu'il était conçu en 2012 ne permettait pas de faire progresser les plus faibles scolairement ${ }^{3}$. Une étude montre qu'il y a une corrélation entre le niveau acquis à l'entrée en $6^{\mathrm{e}}(11$ ans) et la réussite à l'université ${ }^{4}$. A partir de la $4^{\mathrm{e}}$, ces élèves sont devant des devoirs que, quels que soient leurs efforts, ils ne peuvent pas réussir. En effet, ces devoirs demandent des compétences en expression qu'ils n'ont pas et qui ne peuvent pas se rattraper en quelques heures en petit groupe par semaine. Il faut accompagner un effort continu et suivi toute la semaine. Or les cours et les contrôles au niveau collège sont conçus pour faire progresser la première moitié de la classe. Le dogme imposé est que les jeunes rattraperont avec un peu de soutien lorsqu'ils seront prêts à faire des efforts. Il s'agit d'une illusion pour celles et ceux qui ont des fortes lacunes en lecture et en expression, ou pour celles et ceux qui ont des difficultés d'apprentissage spécifiques. Ces élèves arrivent en $3^{\mathrm{e}}$ en ayant perdu la confiance en leur capacité de réussir des exercices scolaires. Peu d'entre eux arriveront à rattraper les acquis de leurs camarades par la suite, le système scolaire ne prévoyant le soutien nécessaire à aucun niveau.

\section{L'orientation par élimination progressive sur le Français}

Le dispositif d'orientation du système éducatif français commence par une élimination progressive par les acquis en Français dans les trois filières du lycée : professionnelle, technologique, générale. En fin de $3^{\mathrm{e}}$, celles et ceux qui n'ont pas les acquis en expression littéraire nécessaires au lycée général et technologique doivent choisir leur filière du lycée professionnel. Puis un deuxième palier se situe à la fin de la seconde. Les élèves qui n'ont pas les acquis en expression littéraire pour réussir les filières générales sont dirigés vers les filières technologiques. Puis les $40 \%$ des lycéens qui ont eu le droit à la filière générale sont triés sur leur niveau en mathématiques. Les causes sont mécaniques. L'expression littéraire en premier lieu et les mathématiques en second sont des fondamentaux indispensables pour la suite des études. Ces compétences s'apprennent dans la durée. La réussite est conditionnée à la mise

\footnotetext{
${ }^{2}$ Colmant \& Le Cam, 2017

${ }^{3}$ Ben Ali \& Vourc'h, 2015

${ }^{4}$ Brinbaum, Hugrée \& Poullarec, 2018
} 
en place de bons automatismes cognitifs. Comme les passerelles et les modules de rattrapage des acquis manquants sont insuffisants dans le supérieur, les élèves stratégiques pour leur avenir prennent la filière qui a le plus haut niveau d'exigence sur les deux ensembles de compétences (expression littéraires et mathématiques) chaque fois qu'ils ou elles le peuvent.

En France, l'élève choisi un domaine à approfondir après la Seconde, comme SES (Sciences Economique et Sociale), STG (Sciences et Technologies de la Gestion), que je nomme dominante, bien que ce terme ne soit utilisé en France. Or chacune de ses dominantes est liée à un niveau d'exigence en expression littéraire et en mathématiques. Comme ces niveaux détermineront les possibilités d'études par la suite à cause du manque de passerelles, cela crée mécaniquement une hiérarchie des « dominantes » du lycée générale et technologique.

Par ailleurs, un double mécanisme entraine la dévalorisation des filières professionnelles. Le premier mécanisme est que seuls les élèves qui n'ont pas les acquis en expression littéraire nécessaires au lycée général et technologique préparent leur orientation, car pour les autres le premier palier d'orientation se situe un an plus tard. Le deuxième mécanisme est que l'orientation se fait par un logiciel qui valorise les notes plutôt que la motivation vers le métier visé. Cela crée une hiérarchie implicite des filières professionnelles basée sur les notes du dernier admis qui se renforce mécaniquement. Les élèves qui ont choisi le professionnel voient celles et ceux qui vont vers la voie que la société considère comme normale, détendus alors qu'ils ont le stress du choix du métier. Bien souvent, ils arrivent dans une filière qu'ils n'ont vraiment pas choisie.

Les dysfonctionnements sont en place depuis la réforme du lycée de 1995 ou depuis la mise en place du logiciel AFFELNET qui sert à l'affectation des élèves pour la voie professionnelle.

\section{LES CRISES DU SYSTEME EDUCATIF FRANÇAIS}

Des crises ont secoué le système éducatif français. Des petites et des grandes crises, certaines sans lendemain. Certaines ont fait la une des journaux, d'autres sont connus uniquement par celles et ceux qui en ont été témoin. Une des crises les plus graves a été l'expression du mal-être chez les enseignants qui a suivi la réforme de la filière STI2D (Sciences et Technologies de l'Industrie et du Développement Durable) de 2010. Elle ne sera pas traitée dans cet article par manque d'informations sur les solutions mises en place. Ces informations n'ont pas atteint le grand public. Aucun travail de sociologues n'a été publié sur cette crise. On peut légitimement se demander si cette absence d'intérêt n'est pas liée au fait qu'il s'agit d'une filière technologique et non pas d'une filière générale.

Le choix est de s'attacher aux crises qui sont liées aux dysfonctionnements décrits dans ce document. Six de ces crises sont représentatives de mécanismes systémiques qui expliquent les blocages du système Education ou ont eu un rôle dans les évolutions depuis 2012 sous les présidences de la République de François Hollande (2012-2017) et d'Emmanuel Macron (depuis 2017).

\section{Le rapport Legrand (1982)}

Le collège unique a été décidé par la loi Haby de 1975. Cependant, dès sa mise en place, des difficultés sont apparues à cause de l'hétérogénéité des classes. Le collège avait une double mission préparer une partie des élèves au lycée général et technologique et faire progresser chaque élève. Le système éducatif a échoué de s'adresser à tous les élèves.

En décembre 1982, à la demande du ministère de l'éducation nationale, Louis Legrand a publié un rapport intitulé «Pour un collège démocratique $»^{5}$. Ce rapport constate les difficultés du collège et propose des solutions innovantes qui ne seront que partiellement mis en œuvre à cause de l'opposition des syndicats d'enseignants.

Le rapport a provoqué de vives polémiques. Les constations réalisées sont très proches de celles actuelles décrites dans le paragraphe «l'abandon structurel au collège de celles et ceux qui n'ont pas les acquis en lecture ». Les élèves en grandes difficultés, comme actuellement, étaient majoritairement issus de milieu défavorisés.

\footnotetext{
${ }^{5}$ Legrand, 1982
} 
Les rapports qui ont suivi ont tous fait état de difficultés très proches. Les tentatives de réformes avant celle de 2015 n'ont pas eu réellement d'effets. Il est trop tôt pour faire un bilan de la réforme de 2015 d'autant plus qu'elle a été dénaturée par l'alternance politique de 2017. Aussi ce rapport, qui date maintenant de 36 ans, décrit des dysfonctionnements qui sont toujours d'actualités.

\section{La mode des méthodes globales de 1980 à 1990}

Dans les années 1970 à 1990, la manière qu'il était politiquement correct de promouvoir pour améliorer l'efficience de l'école consistait à rechercher la méthode innovante, et donc forcément efficace car moderne, que tous les enseignants devraient appliquer. Dans approches testées, il y a eu les méthodes globales en lecture et en calcul que les services centraux ont tenté d'imposer avec plus ou moins de volonté et de succès. Les conséquences sont racontées dans un livre publié en 2004 par Marc Le Bris ${ }^{6}$. En réalité, ces méthodes n’ont jamais été généralisées et les méthodes utilisées après 1990 pouvaient avoir quelques traces issues des méthodes globales, mais avaient un fondement basé sur les approches syllabiques.

Cependant, ce sujet a longtemps alimenté un débat très artificiel entre les dits-Républicains et les ditsPédagogues où chaque groupe caricaturait l'autre. Curieusement, le débat s'est poursuivi alors que celui-ci n'avait pas plus lieu d'être. En 2016, le livre " Qui sont les assassins de l'école ? » décrivait les avancées depuis 2012 et montrait que les difficultés avaient bien été identifiées et qu'une démarche était en place pour trouver des solutions, Il comprenait quelques chapitres qui semblaient être rajoutés, reprenant les poncifs de la querelle des Républicains et des Pédagogues. Ce sont les arguments de ces derniers chapitres qui se sont retrouvés dans le débat du grand public ${ }^{7}$.

Dès le début de la Présidence de François Hollande, en juillet 2012, le projet «LireÉcrireCP » est lancé, aussi appelée étude Goigoux du nom de son coordinateur. Il s'agit d'une étude de grande ampleur impliquant de nombreux chercheurs dans toutes la France dont certains avaient été parties prenantes de la guerre des méthodes. Dans cette étude, les pratiques réelles des enseignantes et enseignants pour l'apprentissage de la lecture et de l'écriture étaient analysées, en particulier pour les élèves d'origine sociale défavorisée ${ }^{8}$. Plus que le résultat qui a confirmé ce que les études sérieuses disaient depuis longtemps, l'apport de cette démarche fut la construction dans la communauté des enseignants-chercheurs en pédagogie d'une connaissance commune sur les bonnes pratiques pour mener une action de recherche et sur ce qui était réellement efficace pour apprendre à lire et à écrire. Le nouveau paradigme a été diffusé par des formations ou des conférences de consensus, le plus souvent relayées sur Internet.

\section{Les émeutes des banlieues de 2005}

En octobre et novembre 2005, les banlieues françaises se sont enflammées. A Clichy-sur-Bois, le 27 octobre, deux adolescents poursuivis par la police se cachent dans un transformateur haute-tension et meurent électrocutés. Les versions des témoins et de la police étaient différentes. La police a réagi en intensifiant la répression. Le mépris ressenti dans les banlieues a déclenché une escalade de violence, qui a connu son apogée la nuit du 6 au 7 novembre où 274 communes sont touchées par des émeutes dans toute la France. Les émeutes n'étaient pas le fait de quelques voyous mais l'expression d'un rasle-bol d'une population'.

La parole des habitants des banlieues s'est libérée. Ils ne se sentaient pas comme ayant les mêmes droits et les mêmes possibilités que les autres Français. L'une des questions centrales étaient les tensions entre la police et les jeunes. Cependant l'hypocrisie du système scolaire, les discriminations dans l'accès à l'emploi faisaient également partie des motifs d'exaspération, d'autant plus que ces injustices ressenties étaient tues voire niées.

\footnotetext{
${ }^{6}$ Le Bris, 2004.

${ }^{7}$ Barjon, 2016.

${ }^{8}$ Goigoux \& al., 2016.

${ }^{9}$ Le Gaoziou \& Mucchielli, 2016.
} 
Ces émeutes et les paroles médiatisées ont mis le sujet de l'égalité des chances à l'ordre du jour. En 2006, l'égalité des chances a été déclaré « grande cause nationale » et les dispositifs de soutien pour la réussite des jeunes de ces quartiers se sont développés. Ils ont été d'une efficacité modérée puisque le sujet est toujours d'actualité. Cependant, des jeunes ont pu en bénéficier et peuvent être à la fois des modèles, des témoins et des relais pour les actions à venir.

Ces dispositifs ont apporté une connaissance sur le sujet aux sociologues et à la société civile impliquée. Depuis quelques années, les communications sur les inégalités montrent une meilleure compréhension des mécanismes complexes et systémiques qui en sont à l'origine. Il a été possible d'expliquer l'illusion de la méritocratie. Beaucoup de ceux et celles issus des parcours les plus sélectifs pensaient que leur réussite était due à leur seul mérite. Ils n'avaient pas conscience des biais liés aux origines sociales et aux lieux d'habitation. Si la France permet à tous de présenter les mêmes concours, l'accès à un entraînement de qualité et l'accompagnement pour être dans les dispositions d'esprit de la réussite ne sont pas identiques pour tous ${ }^{10}$.

\section{Les études PISA}

PISA $^{11}$ est une enquête de comparaison internationale de l'OCDE se déroulant tous les trois ans depuis 2000 et concernant les jeunes de 15 ans. La France est dans la moyenne de l'OCDE. Les performances des élèves sont stables dans le temps. Cependant, la France est un des pays qui atténuent le moins les différences dues aux origines sociales ${ }^{12}$.

Certains pays comme l'Allemagne ont su comprendre les causes des résultats moyens et réagir. Ce n'est pas le cas de la France et ce n'est pas faute d'avoir essayé.

Concomitamment à la première enquête en 2001, le ministre de l'Education Nationale de l'époque, Jack Lang avait publié un document d'orientation pour le collège intitulé "Pour un collège républicain » où il proclamait « Il ne suffit pas de démocratiser l'accès au collège, il faut démocratiser la réussite au collège ». Puis après une alternance politique, son successeur Luc Ferry a fait de même en 2002 avec un document intitulé «Remettre l'école sur le chemin du progrès ». En 2003-2004, s'est déroulé un "grand débat sur l'avenir de l'école» au sein d'une commission présidée par Claude Thélot. Du temps a été libéré dans les établissements pour des groupes de travail impliquant toutes les parties prenantes de l'établissement autour de thèmes comme " Comment prendre en charge les élèves en grande difficulté ? » ou «Comment motiver et faire travailler efficacement les élèves ? Les comptes rendus détaillés ${ }^{13}$ des séances d'un collège des Hauts-de-Seine relatent des échanges qui auraient pu avoir lieu dix ans plus tard. Le rapport ${ }^{14}$ de cette commission mettait en avant le socle commun de connaissances, de connaissances, de compétences et de règles de comportement et proposait de faire vraiment réussir tous les élèves. Ce sont aussi les orientations de la loi de refondation de l'école de 2013.

Depuis, chaque enquête PISA est commentée dans la presse généraliste. Cependant, les réformes qu'elles déclenchent n'ont pour l'instant pas permis de faire évoluer le système Education vers plus d'efficacité.

\section{La présidence de Nicolas Sarkozy (2007-2012)}

Le président de la République Nicolas Sarkozy s'était engagé de réduire les effectifs de la fonction publique. L'école n'a pas été épargnée y compris pour les adultes qui étaient en contact avec les élèves. Ce sont 80000 postes sur un effectif d'environ 1 million de personnes qui ont été supprimés dans l'éducation nationale alors que la population d'élèves ne diminuait pas. En 2007, la France était déjà un des pays de l'OCDE qui avait le plus bas taux d'enseignants par élève en école primaire (élèves de 6 à 11 ans). Pourtant, il est fondamental pour l'efficacité d'un système éducatif que, au

\footnotetext{
${ }^{10}$ Gignoux-Ezratty, 2018.

${ }^{11}$ PISA (Programme International pour le suivi des élèves).

12 Bourny \& al, 2013 et Fumel \& al, 2016.

${ }^{13}$ Document des archives personnelles de l'auteure.

14 Thélot, 2004.
} 
moment du premier apprentissage, l'enfant mette en place des automatismes de lecture efficaces. Pour cela, l'enseignant doit pouvoir avoir une attention à chaque élève lors de ces apprentissages cruciaux.

Les stratégies ont été :

- la suppression de la formation à la pédagogie des enseignants (IUFM ${ }^{15}$ );

- l'augmentation du nombre d'élèves par classe ;

- la diminution du nombre de cours en demi-groupe ;

- la diminution du nombre d'enseignants chargés des remplacements en cas d'absence ;

- la diminution des redoublements sans mise en place de moyens pour aider à rattraper les lacunes ;

- la diminution des moyens pour la remédiation comme les RASED (Réseaux d'aides spécialisées aux élèves en difficultés).

Les conditions d'enseignements qui étaient déjà mauvaises dans certains établissements depuis des dizaines d'années, se sont dégradées. De jeunes enseignants, ayant un master, mais sans formation à la pédagogie ou à la gestion d'une classe se sont retrouvés devant les classes les plus difficiles. Les enseignants déjà titularisés ne pouvaient pas aller en formation, faute de remplaçants disponibles.

Le gouvernement s'appuyait sur les idées reçues de la société sur l'école pour faire passer ses décisions. La communication était très habile, jouant sur les mots. Par exemple, il annonçait qu'il y aurait deux heures par semaine d'accompagnement personnalisé. Ce qui semble bien, mais il fallait comprendre deux heures pour la classe entière, ce qui fait en moyenne 4 minutes par élèves. Quelques classes à projet sportif ou culturel ont été créées avec 30\% de moyens en plus que nécessaires de manière à organiser des reportages pour les informations télévisées portant sur ce que serait l'école dans les années futures. Pendant ce temps, les autres élèves étaient dans des classes surchargées.

Cette période est liée au début de la possibilité d'expression du citoyen sur internet. Les journaux électroniques ont autorisé les commentaires sur leurs articles. Il devint facile de mettre en place un blog. Les réseaux sociaux ont commencé à se structurer. Ces médias sont devenus un vecteur pour dire la réalité des conditions de travail des enseignants et des enseignantes, pour dénoncer les inexactitudes dans les communications officielles. Ces réactions ont participé à la prise de conscience qui a conduit à la refondation de 2013.

Dès la rentrée 2013, des écoles supérieures ont rouvertes pour la formation des enseignants. D'après les déclarations officielles, 60000 postes ont été créés entre 2012 et 2017.

Aujourd'hui, il y a un consensus dans le pays qu'une formation de qualité des enseignants est indispensable, en particulier sur la manière d'enseigner la lecture avec construction de sens pour des enfants ayant des difficultés d'apprentissage, en particulier sur la manière d'obtenir une ambiance de travail dans sa classe.

Il n'y a toujours pas de consensus sur le niveau d'encadrement optimal des élèves. Combien d'élèves pas classes ? Quels cours devraient être en petits groupes ? De quelle manière doit être pris en compte le contexte ? Certains se réfèrent aux comparaisons internationales. Cependant, tous les gouvernements ne voient pas la nécessité d'adapter les moyens à ce niveau. Les enjeux financiers sont importants pour le budget de la nation.

\section{Les attentats terroristes de janvier 2015}

En janvier 2015, c'est la consternation en France. Une série d'attentats endeuillent la nation. Les dessinateurs et journalistes de la rédaction du journal satirique «Charlie Hebdo » sont assassinés. Certaines des victimes étaient connues, le symbole était puissant. Le pays a ressenti une vive émotion. Plus de 4 millions de personnes ont manifesté lors des «marches républicaines » organisées les 10 et 11 janvier à travers la France dont, le dimanche 11 janvier, 1,5 million à Paris.

\footnotetext{
${ }^{15}$ Institut universitaire de formation des maître (IUFM).
} 
L'école a organisé des discussions dans les classes. La réaction de certains jeunes qui refusaient de s'associer à la minute de silence a sidéré le corps enseignant. Certains ont affirmé leur désaccord ou même leur déni. L'expression des enfants peut être considérée comme une provocation à une injonction d'une institution qui les rejette ${ }^{16}$.

Il y eu au niveau de la nation une prise de conscience que les injustices ressenties dans les territoires ségrégués attaquaient les fondements des valeurs républicaines. Des jeunes qui étaient nés en France et avaient fait toute leur scolarité dans les écoles publiques de la République avait rejeté leur pays au point de commettre des attentats contre lui. Ce fut une raison de mobilisation des personnes impliquées pour l'école de s'attaquer aux blocages de celle-ci.

\section{LES JEUX DE POUVOIRS ENTRE LES COURANTS DEPUIS 1990}

Cette analyse est basée sur les constatations de l'auteure qui est en situation d'observation du système éducatif à divers niveaux depuis 20 ans. Ce paragraphe décrits les courants qui pendant des décennies ont bloqué les changements et absorbé les crises sans rien changer de leur manière d'agir jusqu'à la prise de conscience de 2012.

\section{Des courants qui bloquent les changements dans l'école}

Depuis 30 ans, les personnes s'intéressant à l'école se sont organisées en groupes portant des convictions sur la manière de réformer, ou d'empêcher la réforme de l'école, avec leurs messages pour convaincre et des arguments agressifs contre ceux et ceux qui portent une analyse différente que la leur. Ces groupes ont mis en place des «polémiques artificielles » qui ont pendant longtemps parasité les débats sur l'éducation, au point de les rendre impossibles.

Ces groupes, au nombre de quatre, sont formés d'associations, de syndicats d'enseignants, de communicants en pédagogie, et ont des sympathisants dans les partis politiques ou chez des citoyens et citoyennes impliqués qui s'intéressent à l'école. Ce sont :

- la technostructure de l'Education nationale,

- les autoproclamés-progressifs,

- les élitistes paradoxaux;

- les élitistes assumés.

Ils utilisent tous la même technique pour se différencier et construire artificiellement de l'antagonisme. Sur un sujet complexe, ils proposent une solution contre-productrice mais donnant l'impression que c'est pour l'intérêt commun. La solution est accompagnée d'éléments de langage, dont certains ont pour rôle de justifier la solution et d'autres ont pour rôle de dénigrer celles et ceux qui tendent à rétablir la réalité. Ces éléments de langage ont été relativement stables entre 1990 et 2012.

Il y avait une entente tacite. Lorsqu'un groupe déployait ses dénigrements contre ceux qui n'étaient pas d'accord, les membres des autres groupes se taisaient, et ce, sauf si les enjeux perçus dépassaient un niveau critique.

Lorsque les dirigeants de l'Education Nationale souhaitaient diminuer les coûts de l'Education Nationale, ils négociaient avec les autoproclamés-progressifs, les élitistes paradoxaux et les bureaucrates des services centraux, pour des solutions qui étaient compatibles avec le « fonds de commerce » de chacun.

Il y a une auto-finalisation autour de quatre « ensemble de croyances » avec des moteurs puissants qui a, pendant des décennies, résisté aux crises. Ceci peut être analysé comme un système avec quatre attracteurs et des boucles de rétroaction. Depuis 2012, des évolutions notables ont eu lieu grâce à la mobilisation de la société civile qui croit que l'accès à l'éducation pour tous est un rempart contre le populiste, grâce à internet qui a permis d'échanger sur des sujets où des groupes d'intérêts imposaient des dogmes et grâce au travail de fond fait autour de la refondation de l'école de 2013 qui a accompagné un changement de paradigme chez les autoproclamés-progressifs.

\footnotetext{
${ }^{16}$ Simon A. 2018
} 


\section{La technostructure de l'Education nationale (courant 1)}

La technostructure de l'Education nationale, comprend les dirigeants et les bureaucrates de l'Education nationale : ceux qui créent, loin du terrain, la règle et les programmes informatiques de suivi ou d'affectation.

On observe un mécanisme de bureaucratisation classique bien connu en sciences des organisations depuis les travaux de Michel Crozier. Ceci a été amplifié récemment avec l'apparition de logiciels pour suivre les résultats où régir les affectations des élèves. Les sociologues du travail commencent à documenter les dysfonctionnements qui se mettent en place quand des systèmes informatiques pilotent des activités loin du terrain ${ }^{17}$. Leurs résultats peuvent s'appliquer aux systèmes informatiques de gestion de l'Education Nationale.

La technique utilisée par la technostructure pour conserver le pouvoir était simple. Si un enseignant disait qu'il n'arrivait pas à faire le travail prescrit, on lui répondait qu'il n'utilisait pas le dernier gadget pédagogique à la mode qui était en réalité inefficace. Il comprenait que ce qu'on attendait de lui était de taire les problèmes, de bien répéter les éléments de langage officiels et faire au mieux dans le secret de sa classe. Les syndicats majoritaires se taisaient dans un accord tacite lié au besoin de conserver leur pouvoir.

Parfois, la hiérarchie intermédiaire faisait filtre et créait un environnement de travail constructif déconnecté des communications du cabinet du ministère et des services centraux. Parfois, la solidarité entre les enseignants de l'établissement permettait de trouver des solutions pour faire au mieux malgré les injonctions contradictoires. Parfois ce n'était pas le cas.

L'omerta sur ce qui se passe dans les établissements a été cassée grâce à Internet et les réseaux sociaux. Des enseignants ont pris l'habitude de décrire, sous pseudonyme, les conséquences des injonctions inadaptées et des exemples de manque de soutien inadmissible de la hiérarchie. La prise de conscience liée aux crises décrites dans le paragraphe précédent a fait le reste. Depuis environ 2015, il n'y a plus de paroles agressives quand on décrit ce qui se passe réellement dans les établissements. Ce qui a permis la multiplication des écrits sur ce qui, il y a quelques années, était tabou.

\section{Les autoproclamés-progressifs (courant 2)}

Ce sont les personnes qui veulent faire évoluer l'école et qui se coordonnent pour promouvoir le même modèle. Jusqu'en 2012, ce qui faisait consensus était souvent déconnecté des résultats des études sérieuses, et ne prenait pas réellement en compte les particularités des enfants ayant des difficultés d'apprentissage. Ils ont été longtemps complices de la technostructure de l'Education Nationale en diffusant les dogmes pédagogiques choisis par cette dernière pour servir le jeu de pouvoir décrit dans le paragraphe précédent.

Les personnes qui se sont impliquées pour faire évoluer le système éducatif sous la présidence de Hollande entre 2012 et 2017, se sont appuyées principalement sur ce courant pour créer une dynamique dans l'école. Ils l'ont fait en ayant une stratégie pour contourner les utopies.

La loi de refondation de l'école a été écrite en mars 2013, après une concertation nationale en septembre 2012, et en associant l'opposition parlementaire.

Cette loi confirmait la volonté d'une école inclusive jusqu'à 15 ans, qui donne les fondamentaux qui permettent de trouver sa place dans la société à tous et qui aide chacun et chacune à progresser et s'épanouir.

Elle a créé des instances indépendantes de la technostructure de l'éducation nationale pour animer des réflexions en dehors de l'autorité hiérarchique. Ces instances impliquent des experts du domaine mais aussi la société civile et des parlementaires représentant à parts égales la majorité et l'opposition. Ces deux instances sont le CSP (Conseil Supérieur des programmes) et Cnesco (Conseil national d'évaluation du système scolaire). Il s'agit d'instances ayant un rôle de conseil, la décision finale incombe au ministre.

\footnotetext{
${ }^{17}$ Dujarier, 2015.
} 
Le principal enjeu de la réforme de la refondation de l'école est de faire évoluer les représentations sur l'école de celles et ceux qui bloquent le système c'est-à-dire, la technostructure, les syndicats, les communicants et les personnalités qui sont écoutées par les enseignants et enseignantes.

Parmi les actions qui ont permis le changement, il y a celle autour du projet LireÉcrireCP déjà décrit dans le paragraphe sur la mode des méthodes globales.

Ce courant a une attirance pour les approches constructivismes où le jeune a une démarche active pour construire lui-même sa connaissance. Cependant, aujourd'hui, une majorité d'entre eux reconnaissent que la pédagogie basée sur un enseignement explicite est plus efficace pour l'apprentissage des fondamentaux aux enfants issus de milieux défavorisés, et en particulier pour les enfants issus de famille en grande pauvreté. Des personnes crédibles pour ce courant ont porté et diffusé des résultats d'études portant sur des pratiques réelles ${ }^{18}$. Il y a donc un large consensus pour l'utilisation des méthodes explicites pour la transmission des savoirs fondamentaux.

La dernière évolution et certainement celle qui a le plus d'impacts est ce que les spécialistes appellent l'abandon de la "pensée magique ». C'est un véritable changement de paradigme. Il était admis parmi « la technostructure de l'éducation nationale » et chez « les autoproclamés-progressif » que, pour résoudre les problèmes de l'école, il fallait trouver la méthode pédagogique universelle, que la direction de l'Education Nationale devait imposer à tous les enseignants. Ils étaient dans l'esprit que tout ce qui est innovant était mieux. Un tabou était imposé : il n'était pas politiquement correct de dire le contraire.

Aujourd'hui, il y a un quasi-consensus pour reconnaître que chaque établissement est particulier, chaque classe est particulière et chaque élève est particulier. Aussi, il n'existe pas de méthodes génériques qu'il suffirait d'appliquer. L'enseignant ne doit plus être considéré comme un opérateur qui doit appliquer avec diligence, les consignes venus d'en haut. Il doit être considéré comme un expert de haut niveau qui fait avancer la classe en général et chaque élève en particulier.

Les évolutions dans les représentations de ce groupe sont toujours niées par des personnes de courants concurrents ou de partis politiques traditionnellement en opposition.

\section{Les élitistes paradoxaux (courant 3)}

J'appelle élitistes paradoxaux ceux qui veulent garder un enseignement qui s'adresse à la première moitié de la classe après le CE2 (élèves de 9 ans) et qui imposent de penser le dispositif d'orientation scolaire pour favoriser celles et ceux qui ont acquis des compétences en expression littéraire accessibles que par $40 \%$ de la population. Ils sont à l'origine des mécanismes qui dévalorisent l'enseignement professionnel décrit dans le paragraphe sur l'orientation par élimination progressive sur le français.

Ils se disent pour l'égalité des chances cependant ils s'opposent à un changement d'organisation qui mettrait l'attention jusqu'à la fin du collège sur la réussite des enfants qui ont des difficultés particulières d'apprentissage.

Le but des enseignants et enseignantes proches de ce courant est de pouvoir continuer à transmettre à la tête de classe les connaissances qui les ont passionnées et qui ont fait qu'ils ont choisi ce métier. Ils aident les plus en difficultés dans les limites du temps disponible en sachant pertinemment que, sauf rares exceptions, cela ne suffira pas pour leur permettre de récupérer leurs lacunes. Ce sont des gens réellement impliqués pour la réussite des enfants, mais en se basant sur une forme d'enseignement qui convient surtout à ceux qui ont des facilités d'apprentissage. Pour eux, il suffirait de rajouter des heures, d'avoir des tailles de classes raisonnables et de d'avoir plus d'apprentissages en demi-groupe pour faire réussir tous les élèves. C'est une illusion et il faut procéder autrement pour éviter le découragement.

Ils ont des tactiques pour nier la désespérance de celles et ceux n'ont pas les fondamentaux pour réussir quoi que ce soit, et qui n'ont pas la compréhension du monde des adultes pour se projeter dans le futur. Par exemple, avant la réforme du collège, quand on disait que les contrôles tels que pratiqués

\footnotetext{
${ }^{18}$ Delahaye, 2015.
} 
au collège étaient inappropriés car ils mettaient en échec de manière systématique celles et ceux qui ont des lacunes sur les fondamentaux de lecture et d'expression, leurs activistes nous disaient que nous étions méprisants car nous pensions que les enfants issus d'un milieu modeste sont moins intelligents. Il se trouve que les surdoués sont souvent en échec car ils mettent en place des processus d'apprentissage inappropriés. Cependant, le rattrapage de lacunes incrustées depuis plusieurs années ne se fait pas sans un soutien spécifique continu et un accompagnement par des enseignants ou enseignantes expérimentés et formés à cette problématique.

Ce groupe a été à l'origine des difficultés de la mise en place des réformes du collège successives. Ils sont soutenus par de nombreux parents impliqués pour la réussite des études de leurs enfants. Il s'agit d'un blocage difficile à lever car il est ancré dans les représentations de la société sur la bonne manière de réussir ses études et sa vie. Or le débat sur ce sujet n'a jamais eu lieu.

\section{Les élitistes assumés (courant 4)}

Les élitistes assumés sont ceux qui veulent une diversité de parcours beaucoup plus tôt, dès la $6^{\mathrm{e}}$ pour certains, et revenir à la pédagogie d'autrefois qu'ils ont idéalisée.

Ce courant est très minoritaire chez les enseignants et dans la société civile impliquée, mais il porte des croyances très partagées dans le grand public. En effet, il y a de multiples exemples d'enfants qui se désintéressaient des apprentissages en CM2 et qui, un jour, se sont investis dans le travail scolaire et ont réussi. Les enquêtes PISA montrent que l'organisation qui permet la meilleure efficacité est une « école inclusive » jusqu'à 15 ans.

Ce groupe s'est construit en opposition avec les autoproclamés-progressifs, à l'époque où ces derniers pensaient que l'innovation pédagogique pouvait sauver l'école.

\section{LA CAPACITE ACTUELLE DE L'ECOLE A AFFRONTER LES CRISES}

Que peut-on dire aujourd'hui de la capacité de l'école d'apprendre de ces crises et en affronter de nouvelles?

La question est difficile à cause du manque de recul sur les intentions de ceux qui sont au pouvoir actuellement. Cependant, il est possible de mettre en avant quelques caractéristiques.

\section{Une meilleure compréhension des problématiques par la société civile impliquée}

La société civile impliquée a su apprendre des crises de l'école. Depuis environ 2015, les débats ont changé. Il y a une meilleure compréhension des mécanismes sociologiques qui sont à l'origine des inégalités et une prise de conscience du risque que l'on fait prendre en ne traitant pas les sentiments d'injustice liée à un lieu d'habitation ou à des origines.

Un groupe de personnes de tout bord ont su dépasser les débats stériles pour discuter autour des enjeux de l'école et de la nécessité d'enseigner les connaissances implicites de l'école à celles et ceux qui ne les ont pas par leur famille.

Les informations inexactes sont systématiquement dénoncées sur les réseaux sociaux. Il y a une nouvelle génération de journalistes spécialisées qui vont sur le terrain et ne se contentent pas de recopier les communiqués de presse ou les dogmes d'un des courants décrits plus haut.

\section{Des priorités qui ont traversé les alternances politiques}

Certaines des priorités sont restées malgré l'alternance politique :

- une formation des enseignants adaptée à leur métier;

- la transmission des fondamentaux à tous : lire, écrire, respecter autrui,

- le climat scolaire et la lutte contre les violences scolaires,

- l'inclusion des élèves en situation de handicap,

- la valorisation et le développement de l'enseignement professionnel,

- l'implication des entreprises et des régions pour l'information sur les métiers. 
Il y a une continuité sur des sujets qui font consensus. C'est important car les changements de priorité incessants créent du découragement. Il est difficile de s'investir sur un sujet quand on sait qu'il sera remis en cause pour des raisons de communication à la prochaine alternance politique.

\section{Un manque de moyens humains pour faire face aux enjeux}

Toutes les Françaises et tous les Français ne voient pas l'école comme un investissement pour le pays. Depuis 2007, chaque fois que la droite est au pouvoir, la droite diminue les moyens de l'Education nationale que ses successeurs rétablissent partiellement.

Les comparaisons internationales nous donnent le taux d'enseignants devant les enfants, au niveau primaire, au niveau collège et au niveau lycée général, ainsi que la performance du pays. Pourquoi ne pas s'en servir comme repère ? Lors de l'alternance politique de 2017, les «emplois aidés » prévus à la rentrée ont été annulés. Des enfants porteurs de handicap se sont retrouvés sans l'aide indispensable qui leur permet d'être scolarisés. Les établissements ont dû revoir leur fonctionnement par manque de personnel pour les travaux administratifs et pour la surveillance des couloirs.

Le ministre actuel, Jean-Michel Blanquer a fait une priorité de l'apprentissage de la lecture en milieu prioritaire. Pour cela, il a imposé des très petits effectifs en REP et REP+ (Réseau d'éducation prioritaire) pour les classes d'apprentissage de la lecture (CP et CE1). C'est une action qui a fait l'unanimité. Cependant, cette opération s'est faite sans moyens supplémentaires et aux dépens des autres écoles. Le dernier arbitrage budgétaire a acté une diminution du nombre de postes d'enseignants, certes modérée, mais le symbole est là. Plus de personnels dans les établissements permettent des projets plus ambitieux qui sont abandonnés au moment des restrictions. L'absence de stabilité des moyens crée du désengagement et empêche de capitaliser les bonnes pratiques dans la durée.

\section{Une destruction actuelle des boucles de régulation systémique}

Alors qu'un Conseil Supérieur des Programmes (CSP) a été installé en 2013 et que des personnes qualifiées ont travaillé pour construire des programmes scolaires cohérents du CP à la $3^{\mathrm{e}}$ (élèves de 6 ans à 15 ans) et correspondant aux enjeux d'une loi qui avait fait consensus, le ministre est revenu aux habitudes précédentes. Il décide en petit comité en dehors de tout éclairage indépendant. Le Conseil des programmes a le rôle d'une chambre d'enregistrement. Ce choix peut trouver une explication stratégique quand il s'agit de modifier à la marge les programmes mis en place par les précédents de manière à faire plaisir à des groupes de pression. Cependant le même processus est en train d'être appliqué aux programmes du nouveau lycée pour lesquels une analyse des enjeux serait nécessaire.

Un projet de loi prévoit la suppression du $\mathrm{Cnesco}^{19}$, cet organisme qui analysait les résultats du système éducation de manière indépendante et qui a été moteur pour remettre en place des pratiques efficaces pour la lecture.

Or, le blocage de l'éducation nationale était en grand partie dû à l'habitude des décideurs et de la technostructure de l'Education Nationale d'arranger les études pour appuyer ce qu'ils ont décidé de mettre en place, de faire appel aux chercheurs qui savent mettre en avant ce qu'ils ont envie d'entendre plutôt que celles et ceux qui cherchent à comprendre les moteurs des dysfonctionnements. La motivation était la diminution des coûts ou la mise en valeur de la capacité de réformer du ministre.

Il est trop tôt pour avoir un avis sur la politique d'évaluation. Cependant, les évaluations qui ont déjà été mises en place ne prennent pas les précautions indispensables pour éviter les biais de mesure et d'interprétation spécifiques au cas d'un système complexe. Dans un système complexe, de nombreux éléments, parmi ceux interdépendants et indispensables pour son efficacité, ne sont pas évaluables simplement.

Ce qui semble se dessiner aujourd'hui dans l'éducation est l'abandon de la gouvernance de la construction d'une connaissance collective des parties prenantes du Système éducation, et un retour vers un fonctionnement tayloriste où tout est décidé dans les bureaux des conseillers du ministre. La

\footnotetext{
${ }^{19}$ Cnesco (Conseil national d'évaluation du système scolaire), organisme créé par la loi sur la refondation de l'école de 2013.
} 
systémique nous enseigne que ce type de direction est inadapté aux systèmes complexes. Par ailleurs, c'est ce mode de management qui a permis l'incrustation des dysfonctionnements depuis 40 ans.

\section{CONCLUSION}

Aujourd'hui, l'école française est toujours dans l'expectative. Elle n'est pas à l'abri d'une crise. Les moyens nécessaires pour régler les dysfonctionnements décrits au début de cet article ne sont pas en place. La tactique actuelle du gouvernement s'appuie parfois sur les jeux de pouvoir des courants. La « technostructure » est chargée de transmettre la communication du ministre parfois en désaccord avec la réalité perçue sur le terrain. Les croyances des «élitistes assumées » sont parfois utilisées dans la communication vers le grand public. La dernière réforme du lycée a été décidée par consensus entre les courants, ce qui a toujours conduit à renforcer les dysfonctionnements.

La société civile impliquée a une meilleure compréhension des enjeux, des mécanismes qui créent des inégalités et sur quoi il faut mettre des priorités. Une partie des dogmes portés par les jeux de pouvoir entre les courants qui bloquent le système ont sauté. Cependant des croyances fausses restent. Des «espaces de co-construction» qui permettaient de développer une compréhension commune des problématiques et une expertise indépendante sur les sujets complexes au bon niveau, ont été partiellement détruits depuis l'alternance politique de 2017.

La stabilité dans le futur de ce qui se met en place n'est pas garantie. Les changements de cap systématiques à chaque changement politique depuis 2007 alimenteraient plutôt la croyance contraire. Il ne sera pas possible de rendre plus attractif le métier d'enseignant dans un contexte d'incertitude sur son contenu.

Il n'y a pas de garantie que la politique d'évaluation annoncée n'amènera pas les dérives qui ont été constatées dans d'autres pays qui ont eu une politique analogue : accélération de la ghettoïsation de certains établissements et perte d'efficacité à cause d'une focalisation sur des critères au lieu de chercher à mettre en place une dynamique collective qui permet le soutien à chaque enfant ayant des besoins particuliers. Tout dépendra de la capacité de changer de stratégie à moyen terme avant la réification des indicateurs, forcément partiels, en critères.

L'affichage d'une volonté de revenir aux décisions centralisées et de supprimer les institutions indépendantes chargées de construire une connaissance partagée sur les sujets complexes, est inquiétante.

Dans ces conditions, la société civile a une responsabilité dans l'avenir de l'éducation du pays. Elle doit conserver son rôle de boucle de régulation systémique qui éclaire sur les enjeux, construit une représentation commune qui donne une visibilité à l'ensemble du système, anime des groupes de réflexion sur les sujets cruciaux et alerte sur les risques de reproduction des crises que le système éducatif a déjà traversées.

\section{REFERENCES}

Barjon, C. (2016), Mais qui sont les assassins de l'école ?, Edition Robert Laffont.

Ben Ali, L. \& Vourc'h, R. (2015), "Acquis des élèves au collège : les écarts se renforcent entre la sixième et la troisième en fonction de l'origine sociale et culturelle", Note d'information, $\mathrm{n}^{\circ} 15.25$, MEN-DEPP, août 2015.

Bourny, G., Fumel, S., Keskpaik, S. \& Trosseille B. (2013) "L’évolution des acquis des élèves de 15 ans en compréhension de l'écrit et en culture scientifique - Premiers résultats de l'évaluation internationale PISA 2012" Note d'information, n¹3.30, MEN-DEPP, décembre 2013.

Brinbaum, Y., Hugrée, C. \& Poullaouec, T. (2018), "50 \% à la licence... mais comment ? Les jeunes de familles populaires à l'université en France », Economie et Statistique, 499, 79-105.

Colmant, M. \& Le Cam, (2017), "PIRLS 2016 : évaluation internationale de élèves de CM1 en compréhension de l'écrit - Evolution des performance sur quinze ans", Note d'information, $\mathrm{n}^{\circ} 17.24$, MEN-DEPP, décembre 2017. 
Delahaye, J.-P. (2015), Grande pauvreté et réussite scolaire - Le choix de la solidarité pour la réussite pour tous - Rapport à madame la ministre de l'Education Nationale, et de l'Enseignement supérieur et de la Recherche.

Dujarier M.-A. (2015), Le management désincarné - Enquête sur les nouveaux cadres du travail, Edition de la découverte.

Fumel, S., Keskpaik, S., Salles, F. \& Verlet, I. (2016), "PISA 2015 : L'évolution des acquis des élèves de 15 ans en compréhension de l'écrit et en culture mathématique.", Note d'information, $\mathrm{n}^{\circ} 16.384$, MEN-DEPP, décembre 2016.

Gignoux-Ezratty, V. (2018) «Le déterminisme social et genré dans l'éducation », Actes des Journées d'Andé, AFSCET, le 4-6 mai 2018

Goigoux, R. \& al. (2016) - Lire et Ecrire - Rapport de recherche - Etude de l'influence des pratiques d'enseignement de la lecture et de l'écriture sur la qualité des premiers apprentissages - Sous la direction de Roland Goigoux - Université de Lyon - ifé (Institut Français de l'éducation) - ENS de Lyon.

Le Gaoziou, V. \& Mucchielli, L. (2006), Quand les banlieues brûlent. : Retour sur les émeutes de novembre 2005, Editions La Découverte

Legrand, L. (1982), Pour un collège démocratique - Rapport au ministre de l'Education nationale, La documentation Française, Paris.

Le Bris, M. (2004) Et vos enfants ne sauront plus lire ni compter. - La faillite obstinée de l'école français, Stock

Simon, A. (2018). L'attentat de Charlie Hebdo du point de vue d'élèves dits «musulmans »: Étude sur l'action politique de l'école. Agora débats/jeunesses, 78,(1),23-40.

Thélot, C. (2004), Pour la réussite de tous les élèves, rapport de la Commission du débat national sur l'avenir de l'école, sous la direction de Claude Thélot, La Documentation française. 